\title{
钙钛矿锰氧化物低维纳米结构研究进展
}

\author{
李磊，梁笠智，吴恒，梁爽，朱菜芶，朱信华 \\ (南京大学 物理学院, 固体微结构物理国家重点实验室, 南京 210093)
}

摘 要: 钘钛矿结构镇氧化物由于同时存在电荷、自旋、轨道、晶格等多种自由度, 它们之间很强的相互作用和相 互竞争导致了一系列新颖的物理现象，如庞磁电阻效应、巨磁熵效应、绝缘体-金属转变、电子相分离、电荷/轨道 有序等现象, 使其成为凝聚态物理学研究的热点。随着微电子器件日趋集成化和微型化, 其特征尺寸越来越小, 目 前基于钻钛矿结构锰氧化物微电子器件的特征尺寸已经进入纳米尺度。在纳米尺度钻钠矿结构锰氧化物具有显著 的尺寸效应，表现出与薄膜及块材不同的电、磁输运特性，在新一代微电子器件领域具有重要的应用价值。近年来 人们在铛钛矿镇氧化物低维纳米结构制备、电磁输运特性测量、微结构表征及理论模拟方面, 都取得了较大的研究 进展, 本文对此进行了评述。首先, 概述了钙铁矿镇氧化物低维纳米结构的微结构研究进展; 介绍了钙铁矿镇氧化 物低维纳米结构的电子相分离及电荷有序现象; 评述了其电磁输运特性的纳米尺度表征; 讨论了钻钛矿锰氧化物 低维纳米结构在自旋电子学、磁随机存储器和传感器方面的应用进展。最后指出了未来钻钛矿锰氧化物低维纳米 结构研究需要重点解决的一些问题。

关 键 词: 钻钛矿结构锰氧化物; 低维纳米结构; 物性与微结构表征

中图分类号: TQ174 文献标识码: A

\section{Advances on Low-dimensional Perovskite Manganite Nanostructures}

\author{
LI Lei, LIANG Li-Zhi, WU Heng, LIANG Shuang, ZHU Ying-Ying, ZHU Xin-Hua
}

(National Laboratory of Solid State Microstructures, School of Physics, Nanjing University, Nanjing 210093, China)

\begin{abstract}
In the perovskite-type manganese oxides, multi-degrees of freedom such as charge, spin, orbital and crystal lattice coexist simultaneously, and the strong completing interactions among them result in a series of novel physical phenomena such as colossal magnetoresistance effect, giant magnetic entropy effect, metal-insulator transition, electronic phase separation and charge/orbital ordering, which make them become attractive issues in condensed matter physics. Advances in integration and miniaturization of the electronic devices have resulted in their feature sizes continued to be decreased, and now the feature sizes of electronic devices based on perovskite-type manganese oxides are down-scaled into nanometered sizes. At nanoscale perovskite-type manganese oxides exhibit apparent size effects and possess the electrical and magnetic transport properties that are different from their bulk and film counterparts, which have important applications in the fields of a new generation of microelectronic devices. In recent years, many advances have been made in fabrication, microstructural characterization electrical and magnetic property measurements of the low-dimensional perovskite manganite nanostructures, and also in the
\end{abstract}

收稿日期: 2014-07-14; 收到修改稿日期：2014-09-24

基金项目: 国家自然科学基金(11174122,11134004); 国家科技部重大专项基金(2009ZX02101-4); 江苏省六大人才高峰资 助(XCL-004)

National Natural Science Foundation of China (11174122, 11134004); Key Special Program of the Ministry of Science and Technology, China (2009ZX02101-4); Jiangsu Provincial Six Talent Peaks Fwnding (XCL-004)

作者简介: 李 否(1990-), 男, 硕士研究生. E-mail: lilei7151990@126.com

通讯作者: 朱信华, 教授.E-mail: xhzhu@nju.edu.cn 
theoretical modelling of the resulting properties. In this paper, an overview of the state of art in the low-dimensional perovskite manganite nanostructures is presented. First, the microstructural characterizations of the low-dimensional perovskite manganite nanostructures are reviewed, and then the electronic phase separation and charge ordering phenomena in the low-dimensional perovskite manganite nanostructures are also introduced. Measurements of the electrical and magnetic transport properties at nanoscale are reviewed. Their potential applications in the fields of spintronics, the next-generation magnetic random access memories and gas sensors, are also discussed. Finally, some key problems in the future researches of the low-dimensional perovskite manganite nanostructures are also outlined.

Key words: perovskite-type manganite oxides; low-dimensional nanostructure; characterizations of physical properties and microstructures

钙铁矿结构锰氧化物(化学式为 $\mathrm{RE}_{1-x} \mathrm{AE}_{x} \mathrm{MnO}_{3}$, 其中 $\mathrm{RE}$ 为三价稀土金属元素，如 $\mathrm{La}^{3+} 、 \mathrm{Pr}^{3+} 、 \mathrm{Nd}^{3+} 、$ $\mathrm{Sm}^{3+}$ 或 $\mathrm{Bi}^{3+}, \mathrm{AE}$ 是二价碱土金属元素, 如 $\mathrm{Sr}^{2+}$ 、 $\mathrm{Ca}^{2+} 、 \mathrm{Ba}^{2+}$ 或 $\mathrm{Pb}^{2+}$ ) 具有庞磁电阻 (colossal magnetoresistance, CMR) 效应, 在自旋电子学领域具有 潜在的应用前景。另外, 钙钛矿结构锰氧化物属于 强关联电子体系, 其自旋、电荷、轨道和晶格自由 度之间具有很强的相互作用并且相互竞争，导致复 杂的电、磁物理特质(如金属-绝缘体转变, 电荷、 自旋和轨道有序、电子相分离等现象), 涉及到凝聚 态物理学的许多基本问题, 使其成为凝聚态物理学 的研究热点 ${ }^{[1-4]}$ 。Zener ${ }^{[5]}$ 在 1951 年提出了双交换模 型 (double-exchange model), 定性地解释了 CMR 效 应以及电阻率随掺杂浓度和温度的变化趋势, 但对 其它与 CMR 效应相关的实验结果未能给出合理解 释, 如 $\mathrm{La}_{1-x} \mathrm{Sr}_{x} \mathrm{MnO}_{3}$ 的居里温度理论计算值比实验 测量值大得多; 在居里温度以上的电阻率理论计算 值比实验值低几个数量级 ${ }^{[6]}$ 。因此, 仅用双交换模型 未能很好地解释钙钛矿结构锰氧化物中的 CMR 效 应，还应考虑晶体中其它的相互作用，如由姜-泰勒 效应 (Jahn-Teller, JT) 引起的电子-声子耦合作用。 为了更合理地解释锰氧化物的输运特性, 如铁磁绝 缘相和顺磁金属相的出现、Mn 位掺杂后金属-绝缘 体转变温度远远低于居里温度的现象, 近年来人们 又提出多种理论模型予以补充和完善, 如单轨道模 型、双轨道模型和电子相分离理论。随着半导体集 成电路和纳米科学技术的迅速发展, 微电子器件日 趋集成化和微型化, 其特征尺寸越来越小, 目前基 于钙钛矿结构镇氧化物的电子学器件已经进入纳米 尺度。纳米尺度的钻钛矿镇氧化物具有显著的尺寸 效应, 表现出与一般薄膜(厚度> $100 \mathrm{~nm}$ )及块材不 同的电、磁输运特性, 在新一代微电子器件领域具 有重要的应用价值 ${ }^{[7-8]}$ 。近年来, 人们在钻钛矿锰氧 化物低维纳米结构的微结构表征、电磁输运特性测 量及理论模拟方面, 取得了较大进展, 本文对此进
行综述。

\section{1 钙钛矿锰氧化物低维纳米结构的微 结构表征}

目前, 表征钙钛矿锰氧化物纳米材料微结构的 方法很多, 如 X 射线衍射(XRD)、扫描电子显微镜 $(\mathrm{SEM})$ 、选区电子衍射(SAED)、透射电子显微镜 (TEM) 以及高分辨电子显微镜(HRTEM)。此外, 还 可以利用电子能量损失谱(EELS)和 X 射线能谱分析 (EDS) 来对其化学组份进行分析。

\section{1 钙钛矿锰氧化物纳米晶和纳米岛}

台湾逢甲大学的 Tsay 等 ${ }^{[9]}$ 利用固相法制备了 $\mathrm{La}_{0.7} \mathrm{Sr}_{0.3} \mathrm{MnO}_{3-\delta}$ 粉末，通过 XRD 图谱证实合成的粉末 样品为纯菱方钙钛矿相, 粉末的平均粒径在 $1.5 \mu \mathrm{m}$ 左 右。粉末颗粒的 EDS 组分分析表明 $\mathrm{La}: \mathrm{Sr}: \mathrm{Mn}: \mathrm{O}$ (原 子百分比 $)=16.02: 6.74: 21.67: 55.43$, 可见, 氧元素 的含量明显低于 $\mathrm{La}_{0.7} \mathrm{Sr}_{0.3} \mathrm{MnO}_{3-\delta}$ 的名义化学组成。 Zabaleta 等 ${ }^{[10]}$ 利用化学组织法在 YSZ 单晶补底上制备 了外延 $\mathrm{La}_{0.7} \mathrm{Sr}_{0.3} \mathrm{MnO}_{3}$ 纳米岛, 其纵向尺寸在 10 $40 \mathrm{~nm}$ 之间, 横向尺寸在 $50 \sim 150 \mathrm{~nm}$ 之间, 纳米岛的 横纵比平均约为 4.0 。

\section{2 钙钛矿锰氧化物纳米线(棒、管)}

Datta 等 ${ }^{[11]}$ 利用水热法合成了 $\mathrm{La}_{0.5} \mathrm{Sr}_{0.5} \mathrm{MnO}_{3}$ 单 晶纳米线, 直径在 $20 \sim 50 \mathrm{~nm}$, 长度在 $1 \sim 10 \mu \mathrm{m}$ 之 间。图 1(a) 是纳米线的 SEM 照片, 插图是相应的 XRD 图谱, 从图中可以看到, 所有 XRD 衍射峰均与 四方结构 $\mathrm{La}_{0.5} \mathrm{Sr}_{0.5} \mathrm{MnO}_{3}$ (JCPDS 89-0786)的衍射峰相 吻合, 由此确定纳米线的晶胞参数为 $a=b=0.545 \mathrm{~nm}$, $c=0.770 \mathrm{~nm}$ 。单根纳米线的 TEM 照片如图 1(b) 所 示, 从图中可以清楚地看到, 纳米线粗细均匀, 且表 面光滑无缺陷, 直径约为 $45 \mathrm{~nm}$; 右下角的插图为纳 米线选区电子衍射图谱, 规则的电子衍射斑点表明 纳米线为单晶, 这可由单根纳米线的 HRTEM 

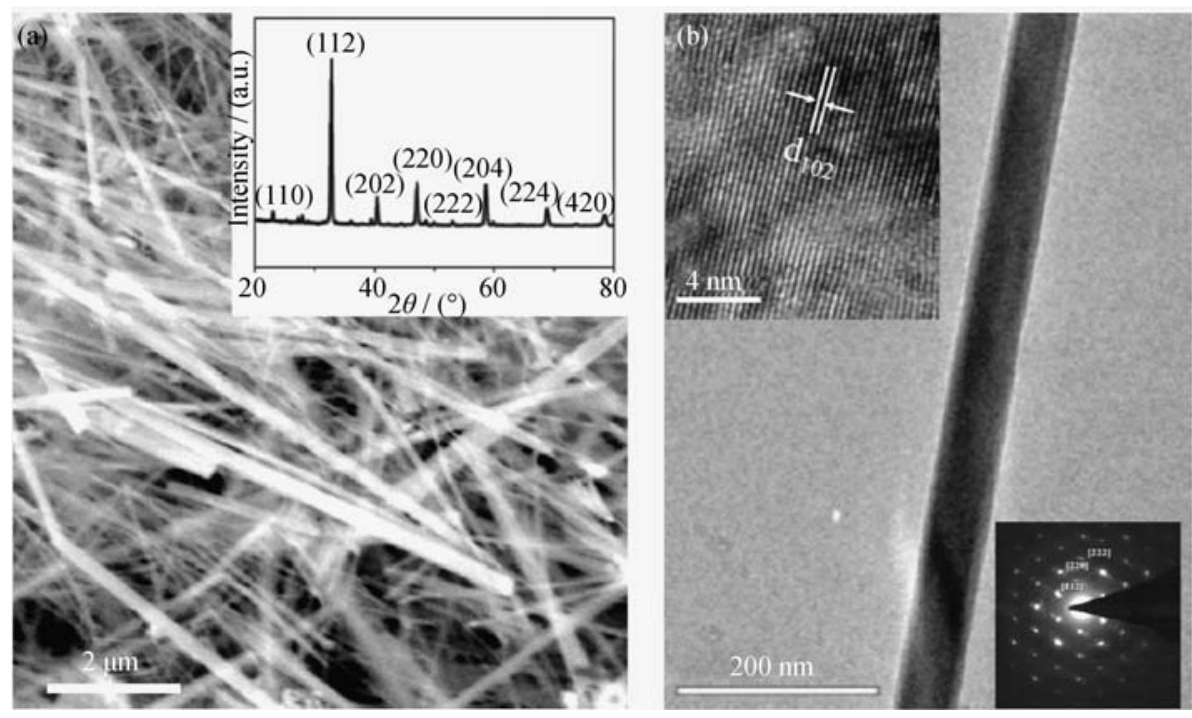

图 1 (a) $\mathrm{La}_{0.5} \mathrm{Sr}_{0.5} \mathrm{MnO}_{3}$ 的 SEM 照片, 插图为其 XRD 图谱; (b) 直径约 $45 \mathrm{~nm}$ 的单根纳米线的 TEM 照片, 左上角插图为 HRTEM 照片, 右下角插图为选区电子衍射图谱 ${ }^{[11]}$

Fig. 1 (a) SEM image of $\mathrm{La}_{0.5} \mathrm{Sr}_{0.5} \mathrm{MnO}_{3}$ nanowires. The inset is the XRD pattern of $\mathrm{La}_{0.5} \mathrm{Sr}_{0.5} \mathrm{MnO}_{3}$ nanowires; (b) TEM image of a single nanowire with a diameter around $45 \mathrm{~nm}$. The inset in the top left corner is the HRTEM image of a single $\mathrm{La}_{0.5} \mathrm{Sr}_{0.5} \mathrm{MnO}_{3}$ nanowires. The inset in the lower right corner is the selected area diffraction pattern taken with $\mathrm{TEM}^{[11]}$

照片(左上角插图)进一步证实。晶面条纹在 HRTEM 像中清晰可见, 条纹间距为 $0.311 \mathrm{~nm}$, 对应于(102)的 面间距。纳米线 EDS 组分分析表明, $(\mathrm{La}: \mathrm{Sr}): \mathrm{Mn}: \mathrm{O}$ 的 原子百分比接近于 $1: 1: 3$, 与 $\mathrm{La}_{0.5} \mathrm{Sr}_{0.5} \mathrm{MnO}_{3}$ 的名义 化学组成相吻合。Banerjee 等 ${ }^{[12]}$ 通过溶胶一凝胶法和 $\mathrm{AAO}$ 模板相结合的方法制备了 $\mathrm{La}_{0.6} \mathrm{Sr}_{0.4} \mathrm{MnO}_{3}$ 纳米管, 平均直径约为 $180 \mathrm{~nm}$ 。Qi 等 ${ }^{[13]}$ 结合溶胶一凝胶法和 熔盐法, 制备出 $\mathrm{La}_{0.7} \mathrm{Sr}_{0.3} \mathrm{MnO}_{3}$ 纳米棒, 平均直径为 $50 \mathrm{~nm}$ ，长度超过 $500 \mathrm{~nm}$ 。

\section{3 钙钛矿锰氧化物低维纳米结构阵列}

2005 年 Ruzmetov 等 ${ }^{[14]}$ 利用激光脉冲沉积、电子束 光刻及氩离子曝光技术相结合, 在 $\mathrm{La}_{0.7} \mathrm{Sr}_{0.7} \mathrm{Al}_{0.65} \mathrm{Ta}_{0.35} \mathrm{O}_{3}$ 单晶祄底上制备了外延 $\mathrm{La}_{2 / 3} \mathrm{Sr}_{1 / 3} \mathrm{MnO}_{3}$ 纳米点阵列, 其三 维 AFM 图像如图 2(a)所示, 图 2(b)为三个代表性纳 米点的 AFM 剖面图, 从图中可以看到, 纳米点阵列
周期性排列, 单个纳米点直径约为 $100 \mathrm{~nm}$, 高度 $37 \mathrm{~nm}$ 。

\section{2 电子相分离现象}

近年来，大量实验研究表明，在钙钠矿结构锰 氧化物体系中存在电子相分离现象, 而且电子相分 离的尺度范围可从纳米量级到微米量级。由于电子 相分离与钲钛矿结构锰氧化物中的 CMR 效应和交 换偏置效应的起源密切相关，而这两种物理效应对 这类材料在自旋电子学器件方面的应用至关重要, 因此，电子相分离成为强关联钙钛矿结构镇氧化物 材料研究中的一个热点问题，揭示其微观物理机制 并实现人为调控对于基础研究和材料的器件化都具 有十分重要的意义 ${ }^{[15]}$ 。以往电子相分离现象的研究主
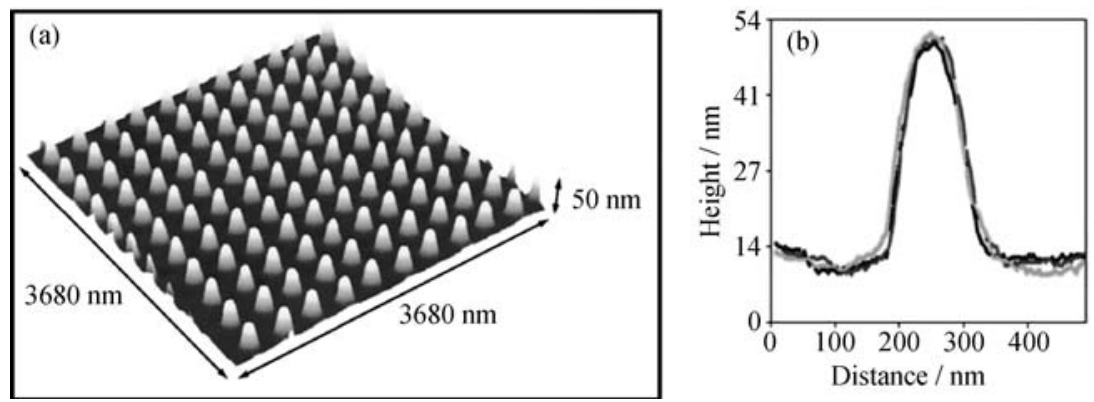

图 $2 \mathrm{La}_{2 / 3} \mathrm{Sr}_{1 / 3} \mathrm{MnO}_{3}$ 纳米点阵列的 $\mathrm{AFM}$ 图像 ${ }^{[14]}$

Fig. 2 Atomic force microscope (AFM) image of a $\mathrm{La}_{2 / 3} \mathrm{Sr}_{1 / 3} \mathrm{MnO}_{3}$ nanodot array ${ }^{[14]}$

(a) 3D AFM image of a $3680 \mathrm{~nm} \times 3680 \mathrm{~nm}$ section of the array. (b) AFM profiles of three representative dots from the AFM image in (a). Dot diameters are $\sim 100 \mathrm{~nm}$, heights are $37 \mathrm{~nm}$ 
要集中在块体和薄膜体系, 近年来钻钛矿锰氧化 物低维纳米结构中的相分离研究日益受到重视, 并取得了一定的进展, 如在 $\mathrm{Pr}_{0.67} \mathrm{Ca}_{0.33} \mathrm{MnO}_{3}$ 纳米 颗粒中, 人们发现了与块材明显不同的相分离演 化行为 ${ }^{[16]}$ 。由于纳米颗粒表面自旋的贡献, $\mathrm{Pr}_{0.67} \mathrm{Ca}_{0.33} \mathrm{MnO}_{3}$ 纳米颗粒在小磁场下呈现出相对 于块体较高的铁磁性。随着磁场的增大, 磁化强度 虽然逐渐增大, 但是没有发生铁磁一金属渗透现象, 直至 $6 \mathrm{~T}$ 也没有出现反铁磁到铁磁的突然转变。这 种现象可归因于表面和核壳界面自旋排列及其自 旋耦合作用的结果。最近, 人们通过磁性测量发现 钙钛矿结构 $\mathrm{La}_{0.33} \mathrm{P}_{\mathrm{r} 0.34} \mathrm{Ca}_{0.33} \mathrm{MnO}_{3}$ 纳米线中也存在 电子相分离现象, 但其相竞争机制与块体明显不同, 表现为纳米线中的磁性冻结相比例有很大的增加 ${ }^{[17]}$ 。 有关钙钛矿锰氧化物低维纳米结构相分离的实验 方面研究进展详见文献[15]。

与此同时, 人们在钻钛矿锰氧化物低维纳米结 构相分离理论计算方面, 也取得一些重要的研究结 果。如 1999 年 Moreo 等 ${ }^{[18]}$ 通过单轨道模型计算, 提 供了相分离模型并描述了铁磁和反铁磁的竞争。进 一步研究发现, 在钻钛矿锰氧化物不同体系不同掺 杂的区域都存在相分离现象, 它与 CMR 效应密切 相关。Yunoki 和 Moreo ${ }^{[19]}$ 对单轨道模型进行了分析, 在局域自旋间引入一个较小的 Heisenberg 耦合后, 发现电子相分离将在较小的电子密度 $(<n>)$ 范围内 出现, 包括铁磁相和未掺杂空穴时的反铁磁相。为 了体现锰氧化物中的轨道有序现象, 人们采用双轨 道模型来描述锰氧化物中的电子相分离。该模型考 虑到体系中 $\mathrm{Mn}$ 位置上的两个不同 $\mathrm{e}_{\mathrm{g}}$ 轨道, 以及 $\mathrm{JT}$ 效应引起的电子-声子耦合效应 ${ }^{[20]}$ 。Yunoki 等 ${ }^{[20]}$ 率 先报道了利用蒙特卡罗方法和双轨道模型获得的锰 氧化物低温相图(1D 情况), 如图 3 所示。该相图包 含了具有轨道序的金属相和绝缘体相。他们同时还 发现由于相分离倾向, 在电子态密度中出现噟能隙 行为。特别重要的是在某些浓度区域, 单一相不能 稳定存在。在低 $\mathrm{e}_{\mathrm{g}}$ 电子浓度区域, 相分离包含了电 子未掺杂时的反铁磁相和金属轨道有序铁磁相。图 3 中虚线表示唯像地认为电子-JT 声子耦合系数随 空穴掺杂下降, 理论上存在的相和实验基本吻合。 另外, 在 $2 \mathrm{D}$ 和 $3 \mathrm{D}$ 情况下人们也得到了类似的计算 结果。因此, 不管采用单轨道模型还是双轨道模型, 都能得到电子相分离结果, 其趋势也很强。进一步 考虑长程库仑作用因素, 可以发现由于库仑相互作 用力的抑制, 不同电荷密度的宏观相分离很难出 现。因此, 两个不同相的大块区域必须分裂成许多 小片, 以使电荷分布更均匀。这样在电子密度不稳
定的掺杂范围内, 长程库仑作用造成一种相的团簇 植入另一相的稳定态。相互吸引的双交换作用和库 仑力之间的相互竞争结果决定了这些团簇的大小和 形状, 如大的液滴和极化子都是最可能的团簇构 型。事实上, 由于考虑库仑作用, 理论计算难度很大, 所以目前还缺乏电荷分布的实际计算结果。在此方 面, 人们还需要更多的努力。

\section{3 电荷有序现象}

电荷有序是指不同带电阳离子在氧化物中特定 晶格位置上的有序排列, 它普遍存在于混合价态的 过渡金属氧化物中, 如在钙钛矿镇氧化物中, 电荷 有序现象与自旋有序、轨道有序、晶格畸变等相互 关联而变得特别引人注目。1996 年 Chen 等 ${ }^{[21]}$ 率先 利用电子衍射技术, 在 $\mathrm{La}_{0.5} \mathrm{Ca}_{0.5} \mathrm{MnO}_{3}$ 接近反铁磁 转变温度 $(95 \mathrm{~K})$ 的电子衍射图谱中发现主衍射斑点 周围出现四个对称等强度的卫星衍射斑点, 它们来 源于 $\mathrm{Mn}^{3+} \mathrm{O}_{6}$ 八面体和 $\mathrm{Mn}^{4+} \mathrm{O}_{6}$ 八面体的有序排列。 1997 年 Radaelli 等 ${ }^{[22]}$ 在 $\mathrm{La}_{0.5} \mathrm{Ca}_{0.5} \mathrm{MnO}_{3}$ 的 XRD 图谱 中也观察到了对称的卫星衍射峰, 同时在其它锰系 氧化物, 如 $\mathrm{La}_{1 / 3} \mathrm{Ca}_{2 / 3} \mathrm{MnO}_{3} 、 \mathrm{Pr}_{1-x} \mathrm{Ca}_{x} \mathrm{MnO}_{3}$ 、 $\mathrm{Pr}_{1-x} \mathrm{Sr}_{x} \mathrm{MnO}_{3} 、 \mathrm{Nd}_{1-x} \mathrm{Ca}_{x} \mathrm{MnO}_{3}$ 和 $\mathrm{Nd}_{1-x} \mathrm{Sr}_{x} \mathrm{MnO}_{3}$ 中都观

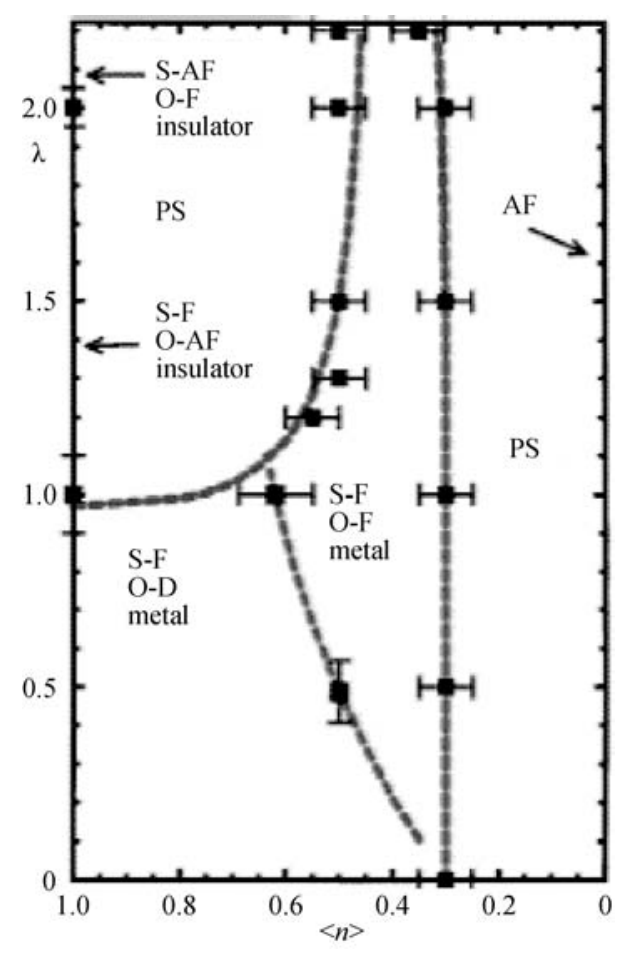

图 3 双轨道模型下 $1 \mathrm{D}$ 相图, 其中 $J_{\mathrm{H}}=8.0, J^{\prime}=0.05$, 跳跃轨 道积分分量 $t_{11}=t_{22}=2 t_{12}=2 t_{21}{ }^{\text {[20] }}$

Fig. 3 Phase diagram of two orbitals model in $1 \mathrm{D}, J_{\mathrm{H}}=8.0, J$, $=0.05$, and using the hopping set $t_{11}=t_{22}=2 t_{12}=2 t_{21}{ }^{[20]}$ 
察到电荷有序现象。1998 年 Mori 等 ${ }^{[23]}$ 利用高分辨 电子显微术在 $\mathrm{La}_{1-x} \mathrm{Ca}_{x} \mathrm{MnO}_{3}(x=1 / 2 、 2 / 3 、 3 / 4 、 4 / 5)$ 体系中观察到电荷有序条纹, 直接证实了该体系在 低温下存在实空间的电荷有序现象。图 4(a)为 $\mathrm{La}_{0.33} \mathrm{Ca}_{0.67} \mathrm{MnO}_{3}$ 的电荷有序条纹像, 从图中可以看 出, 暗条纹的周期为 $3 \mathrm{a}_{0}(=1.65 \mathrm{~nm})$, 它是 $\mathrm{J}-\mathrm{T}$ 畸变的 $\mathrm{Mn}^{3+} \mathrm{O}_{6}$ 八面体条纹。图 4(b)为由图 4(a)中条纹得出 的 $\mathrm{Mn}^{3+}$ 和 $\mathrm{Mn}^{4+}$ 有序排列模型图。对于 $\mathrm{La}_{1-x} \mathrm{Ca}_{x} \mathrm{MnO}_{3}$ 来说, 当 $x=1 / 2 、 2 / 3 、 3 / 4 、 4 / 5$ (分别对应于正交晶胞 $2 / 3 / 4 / 5$ 倍的晶格参数的超晶格结构)时, 电荷有序 现象更易于出现。系统研究结果表明, 电荷有序现 象受多种因素影响, 如掺杂、氧同位素替代和外加 场(磁场、电场、X 射线或等静压)等, 它们可以破坏 电荷有序态, 有关这方面的研究进展详见文献[1]。 Zhang 等 ${ }^{[24]}$ 研究了 $\mathrm{La}_{0.25} \mathrm{Ca}_{0.75} \mathrm{MnO}_{3}$ 化合物不同颗粒 尺寸(40 2000 $\mathrm{nm})$ 下的电荷有序及磁特性, 结果表 明, 当颗粒尺寸减小时, 电荷有序将会由强变弱, 并出现铁磁团簇玻璃态。另外还推论, 当颗粒尺寸 小于临界值 $\left(D_{0}\right)$ 时, 电荷有序态将完全消失。

\section{4 钙钛矿锰氧化物低维纳米结构的物 性表征}

在钙钛矿锰氧化物低维纳米结构中, 由于同时 存在电荷、自旋、轨道和晶格等多种自由度, 而这
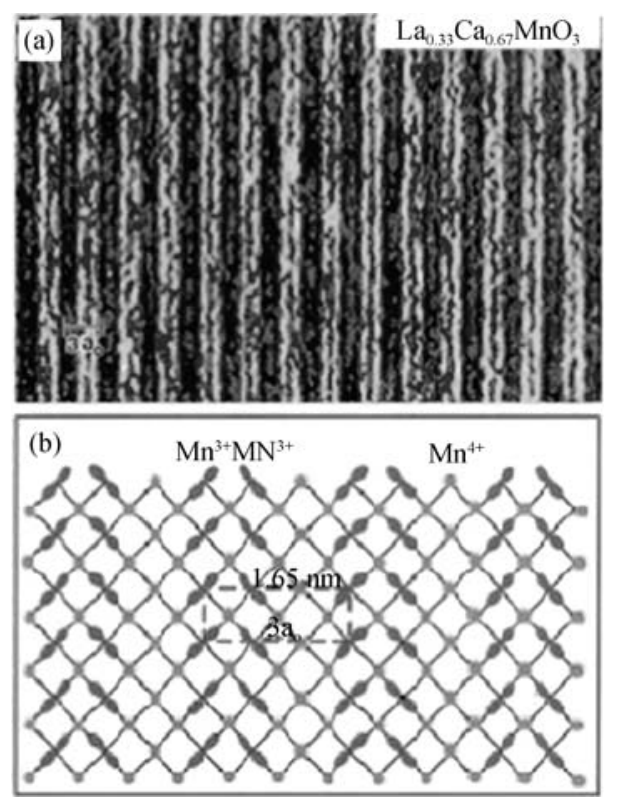

图 $4 \mathrm{La}_{0.33} \mathrm{Ca}_{0.67} \mathrm{MnO}_{3}$ 的电荷有序条纹 ${ }^{[23]}$

Fig. 4 Pairing of charge-ordered stripes in $\mathrm{La}_{0.33} \mathrm{Ca}_{0.67} \mathrm{MnO}_{3}$ (a) High-resolution lattice image obtained at $95 \mathrm{~K}$; (b) Schematic model in the $a-b$ plane showing the pairing and orbital ordering of $\mathrm{Mn}^{3+}$ and $\mathrm{Mn}^{4+}$ [23]
些自由度之间存在很强的相互作用且相互竞争, 在体系中形成一系列能量相近的低能激发态甚至 是相互竞争的基态，在外场作用下呈现出丰富可 控的电、磁输运特性, 在新一代微电子器件领域具 有重要的应用价值, 因此人们对钻钛矿镇氧化物 低维纳米结构的物性进行了大量研究, 取得了如 下进展。

\section{1 钙钛矿锰氧化物纳米晶}

Huang 等 ${ }^{[25]}$ 研究了不同粒径 $\mathrm{La}_{0.25} \mathrm{Ca}_{0.75} \mathrm{MnO}_{3}$ 纳米颗粒的交换偏置(exchange bias)效应, 结果表 明, 其交换偏置场 $\left(H_{\mathrm{EB}}\right)$ 与矫顽场 $\left(H_{\mathrm{C}}\right)$ 会受到颗粒直 径 $(D)$ 的影响, 温度为 $5 \mathrm{~K}$, 颗粒直径为 $80 \mathrm{~nm}$ 左右 时, 交换偏置场与矫顽场最大。近年来, 纳米结构的 钙钛矿镇氧化物光催化特性也引起了人们的关注。

Wang 等 ${ }^{[26]}$ 利用溶胶-凝胶法制备出 $\mathrm{La}_{0.7} \mathrm{Sr}_{0.3} \mathrm{MnO}_{3}$ 纳米颗粒 (晶粒尺寸 20 60 nm), 并对其光催化特性 进行了研究。研究结果表明, $\mathrm{La}_{0.7} \mathrm{Sr}_{0.3} \mathrm{MnO}_{3}$ 纳米颗 粒具有一定光催化活性, 在可见光照射下可降解分 解甲基红, 其光催化机理为光生空穴的直接氧化。

\section{2 钙钛矿锰氧化物纳米线(管)}

相对于薄膜材料而言, 钙钛矿锰氧化物纳米 管、纳米线、纳米棒及纳米纤维以其独特的尺寸效 应及各向异性的物理性质, 日益受到人们的广泛 重视。2011 年, Jugdersuren 等 ${ }^{[27]}$ 利用静电纺丝技术 制备了 $\mathrm{La}_{0.67} \mathrm{Ca}_{0.33} \mathrm{MnO}_{3}$ 纳米线(直径 $80 \sim 300 \mathrm{~nm}$ ), 并在室温下测量了纳米线的磁输运特性, 结果表 明, 室温下 $\mathrm{CMR}$ 效应随着纳米线直径的增加而减 弱; 直径 $80 \mathrm{~nm}$ 的 $\mathrm{La}_{0.67} \mathrm{Ca}_{0.33} \mathrm{MnO}_{3}$ 纳米线在磁场 为 $7.96 \times 10^{4} \mathrm{~A} / \mathrm{m}$ 时的巨磁电阻变化率约为 $28 \%$; 当纳米线直径超过 $280 \mathrm{~nm}$ 时, CMR 效应消失。这 种磁输运行为归因于纳米线内部自旋极化晶粒间 的相互隧穿效应。

近年来, 人们也曾采用物理方法来制备钙钛矿 锰氧化物纳米线。最近 Marín 等 ${ }^{[28]}$ 利用 FIB 技术把 厚度为 $150 \mathrm{~nm}$ 的 $\mathrm{La}_{2 / 3} \mathrm{Ca}_{1 / 3} \mathrm{MnO}_{3}$ 外延薄膜刻蚀成 宽度为 $150 \mathrm{~nm}$ 的纳米线, 研究发现纳米线的电阻 率及其金属-绝缘体相变温度强烈地依赖于纳米线 的宽度; 在磁场很小时(只需 $0.1 \mathrm{~T}$ )磁阻变化可达到 $34 \%$ 。这表明减少纳米线的宽度可以在小磁场下获 得大磁阻效应。

\section{3 钲钛矿锰氧化物薄膜材料}

Jiang 等 ${ }^{[29]}$ 研究了不同厚度 $\mathrm{La}_{0.325} \mathrm{Pr}_{0.3} \mathrm{Ca}_{0.375} \mathrm{MnO}_{3}$ 薄膜的磁性与电阻各向异性, 结果表明, 对于 $30 \mathrm{~nm}$ 厚 的薄膜, 其各向异性电阻率(anisotropic resistivity, AR) 非常大 $\left(\sim 10^{5} \%\right)$; 对于 $120 \mathrm{~nm}$ 厚的薄膜, 由于应变弛豫, 
它的 AR 最大值显著降低 $\left(\sim 2 \times 10^{3} \%\right)$ 。Nori 等 ${ }^{[30]}$ 利用脉 冲激光沉积法在 $\operatorname{Si}(100)$ 上制备了晶粒尺寸为 $20 \sim 60 \mathrm{~nm}$ 的 $\mathrm{La}_{0.7} \mathrm{Sr}_{0.3} \mathrm{MnO}_{3}$ 薄膜, 并对其磁性能进行了表征。结 果表明, 晶粒致密排列的 $\mathrm{La}_{0.7} \mathrm{Sr}_{0.3} \mathrm{MnO}_{3}$ 薄膜居里温度 为 $349 \mathrm{~K}$, 而柱状晶粒组成的薄膜居里温度为 $355 \mathrm{~K}$, 这是目前已报道的沉积在 $\mathrm{Si}$ 衬底上 LSMO 薄膜最高的 居里温度。

最近, 人们对纳米结构的钙钛矿锰氧化物薄膜 的电磁输运特性亦进行了研究, 如 Hirooka 等 ${ }^{[31]}$ 利 用原子力显微镜(AFM)辅助化学湿法刻蚀技术制备 了 $(\mathrm{La}, \mathrm{Ba}) \mathrm{MnO}_{3}$ 纳米桥结构, 发现纳米桥的磁电阻 呈现 2 个数量级的变化。近年来, Guo 等 ${ }^{[32]}$ 采用 $\mathrm{EBL}$ 技术制备了不同宽度的 $(\mathrm{La}, \mathrm{Ca}) \mathrm{MnO}_{3}$ 薄膜微桥结 构, 电输运特性测量结果表明: 薄膜微桥宽度在 $0.50 \mu \mathrm{m}$ 以下, M-I 相变消失, 并且出现反常的正磁 电阻现象。

\section{4 钙钛矿锰氧化物纳米岛}

$\mathrm{Kim}$ 等 $^{[33]}$ 利用脉冲激光沉积法以及 $\mathrm{EBL}$ 技术制备 160 720 nm 尺度的矩形和长六边形 $\mathrm{La}_{0.7} \mathrm{Sr}_{0.3} \mathrm{MnO}_{3}$ 纳米岛。通过控制纳米岛的形状、横 纵比及晶体取向, 观察到了畴态演变。实验结果与 OOMMF (object-oriented micromagnetic framework) 模拟都表明, (110) 取向 $\mathrm{La}_{0.7} \mathrm{Sr}_{0.3} \mathrm{MnO}_{3}$ 长六边形纳 米岛在稳定单畴态方面表现更好, 这在纳米级自旋 电子器件方面具有很好的应用前景。

\section{5 钙钛矿锰氧化物低维纳米结构的 应用}

在钙钛矿锰氧化物低维纳米结构中, 由于同 时存在电荷、自旋、轨道、晶格等多种自由度的关 联与耦合, 从而导致复杂的物理相图和丰富的电、 磁、光等多种物理特性, 使得钙钛矿锰氧化物低维 纳米结构在自旋电子学、高密度信息存储器以及高 灵敏度传感器等领域具有极为广泛的应用, 下面 分别介绍。

\section{1 自旋电子学}

Jin 等 ${ }^{[34]}$ 研究发现, 在磁场和激光作用下, $\mathrm{La}_{0.8} \mathrm{Sr}_{0.015} \mathrm{MnO}_{3}$ 薄膜表现不同的自旋输运特性: 在 整个测试温度区间内, 磁场使薄膜电阻变小, 在 $243 \mathrm{~K}$ 下, 获得最大磁电阻变化率 $(21 \%)$; 激光辐照 薄膜, 在铁磁金属态导致电阻增大, 而在顺磁半导 体态则导致电阻减小, 这主要归因于 $\mathrm{e}_{\mathrm{g}}$ 电子的不同 状态。外加磁场使 $\mathrm{e}_{\mathrm{g}}$ 电子轨道再次简并, 双交换作 用增强, 从而使电阻减小。而激光作用激发自旋向
下 $\mathrm{e}_{\mathrm{g}}$ 电子的跃迁, 改变材料自旋极化方向, 引起光 致退磁现象, 使得金属态的光致电阻增大, 绝缘态 的光致电阻减小。在 $\mathrm{La}_{0.7} \mathrm{Sr}_{0.3} \mathrm{MnO}_{3}$ 薄膜中也观察到 类似的实验现象 ${ }^{[35]}$ 。因此, 利用光场作为外激励手 段, 可改变钲钛矿镇氧化物低维纳米结构体系中的 电子自旋态, 从而影响体系的耦合状态, 改变体系 的物理性能, 这使得钙钛矿锰氧化物低维纳米结构 在自旋电子学方面具有诱人的应用前景。

\section{2 非易失性存储器}

非易失性存储器的优点是即使在断电情况下, 存储的数据也不会随之消失。钙钛矿锰氧化物具有 庞磁阻效应与隧穿磁阻效应, 人们通过改变铁磁层 间的相对磁化方向，可以改变系统的电阻，制作磁 存储器。这种存储器具有非易失性, 目前已经广泛 应用，具有很诱人的商业价值。Liu 等 ${ }^{[36]}$ 按照 $\mathrm{La}_{0.7} \mathrm{Sr}_{0.3} \mathrm{MnO}_{3}$ 的化学计量比, 利用射频磁控溅射方 法在 $\mathrm{Pt} / \mathrm{Ti} / \mathrm{SiO}_{2} / \mathrm{Si}$ 祄底上制备出 LSMO 薄膜, 并发 现 Ag/a-LSMO/Pt 堆垛结构的器件呈现出非易失性 双极电阻开关特性 $\left(\mathrm{R}_{\mathrm{OFF}} / \mathrm{R}_{\mathrm{ON}}>10^{2}\right)$ 、牢固的读/擦耐 久性( $>10^{2}$ 周期)以及长记忆时间(室温下 $\left.>10^{4} \mathrm{~s}\right)$ 。这 些特性在非易失性存储器领域具有重要的应用价 值。Hoffman 等 ${ }^{[37]}$ 系统地研究了 $\mathrm{La}_{1-x} \mathrm{Sr}_{x} \mathrm{MnO}_{3}$ 、 $\mathrm{La}_{1-x} \mathrm{Ca}_{x} \mathrm{MnO}_{3}$ 及 $\mathrm{La}_{1-x} \mathrm{Sr}_{x} \mathrm{CoO}_{3}$ 锰基钻铁矿结构铁电 场效应器件的性能, 结果表明: 电路 RC 时间常数 将器件的开关速度限制到 $80 \mathrm{~ns}$, 这可以实现更快操 作。另外, 室温下运行 $21 \mathrm{~d}$ 后, 器件仍保留了超过 $75 \%$ 的初始开/关比。这些数据都表明该器件在非易 失性存储器领域具有良好的应用前景。

\section{3 传感器}

目前传感器在工业、商业、科研等领域发挥了 非常重要的作用。对于钙钛矿锰氧化物来说, 它具 有庞磁阻效应, 磁电阻变化率大, 在磁传感器领域 具有广泛的应用前景, 如应用于汽车行业中的 ABS 防抱死刹车系统, 还可以通过传感器测量地磁场来 确定船舶或汽车的航向, 这些都是䥻钛矿锰氧化物 在传感器方面的成熟应用。 $\mathrm{Hu}$ 等 ${ }^{[38}$ 利用喷雾热分解 法, 以 $\mathrm{La}\left(\mathrm{NO}_{3}\right)_{3} \cdot 6 \mathrm{H}_{2} \mathrm{O} 、 \mathrm{Sr}\left(\mathrm{NO}_{3}\right)_{2}$ 和 $\mathrm{Mn}\left(\mathrm{NO}_{3}\right)_{2} \cdot 6 \mathrm{H}_{2} \mathrm{O}$ 为 原料, 制备出粒径 $0.3 \sim 2 \mu \mathrm{m}$ 的 $\mathrm{La}_{1-x} \mathrm{Sr}_{x} \mathrm{MnO}_{3}(x=0$ 、 $0.3 、 0.5 、 0.7)$ 粉体, 并研究了其对 $\mathrm{NO}_{x}$ 气体的吸附 和响应特性。当氮氧化物的浓度变化时, 传感器的 响应电流也随之变化, 从而起到动态监测作用。其 中, $\mathrm{La}_{0.5} \mathrm{Sr}_{0.5} \mathrm{MnO}_{3}$ 的吸附特性、线性度、动态响应 性能和敏感性最好, 并且有着较好的抗干扰能力和 较宽的温度范围, 有望作为传感器应用于监控柴油 机排气中的 $\mathrm{NO}_{x}$ 气体浓度。 


\section{6 结束语}

本文对钙钛矿镇氧化物低维纳米结构的微结构 表征、电子相分离和电荷有序现象、物性测量及其 应用领域的研究进展进行了评述。钙钛矿锰氧化物 低维纳米结构以其物理特性以及在自旋电子学、非 易失性存储器和传感器等应用方面所展现出的诱人 商业价值, 受到人们的广泛关注。但是, 仍然存在一 些基础问题需要解决。例如, 尽管人们在钻钛矿结 构锰氧化物不同体系不同掺杂区域中都观察到电子 相分离现象, 但是电子相分离的微观物理本质及其 宽的分离尺度范围 (从几个纳米至微米尺度), 以及 它与 CMR 效应之间的关系, 到目前为止还没有统 一的认识。在钙钠矿锰氧化物低维纳米结构的理论 模拟方面相关的研究还比较少, 目前大多集中在利 用实验手段验证低维纳米材料所表现出的物性与块 体材料的异同。另外, 在钙钛矿锰氧化物在低维纳 米结构的空间几何尺寸接近体系的电子相分离尺度 时, 钙钛矿锰氧化物低维纳米结构中局域物性的变 化可引发体系宏观物性的显著改变, 一些新型物理 效应，如物性层展现象(与电子相分离相关)将被发 现，这对开发以量子效应为基础的新一代钻钛矿锰 氧化物量子调控器件具有十分重要的应用价值, 是 一个值得关注的研究方向。

\section{参考文献:}

[1] CHEN C X. Charge ordering in the perovskite manganites. Journal of Inorganic Materials, 2005, 20(1): 1-12.

[2] HAN L A, MOU G D, HE Y J. Research progress in phase separation in perovskite-type manganese oxides. Materials Review, 2008, 22(z1): 310-313.

[3] LIU Y K, YIN Y W, LI X G. Colossal magnetoresistance in manganites and related prototype devices. Chinese Physics B, 2013, 22(8): 087502-1-19.

[4] LIU P, PENG Z S. Research in phase separation of perovskite-type manganese oxides. Journal of Suzhou College, 2009, 24(1): $107-110$.

[5] ZENER C. Interaction between the d-shells in the transition metals. II. Ferromagnetic compounds of manganese with perovskite structure. Physical Review, 1951, 82(3): 403-405.

[6] MINIS A J, SHRAIMAN B I, MUELLER R. Dynamic Jahn-Teller effect and colossal magnetoresistance in $\mathrm{La}_{1-x} \mathrm{Sr}_{x} \mathrm{MnO}_{3}$. Physical Review Letters, 1996, 77(1): 175-178.

[7] TOKURA Y. Correlated-electron physics in transitional-metal oxides. Physics Today, 2003, 56(7): 50-55.
[8] DAGOTTO E. Complexity in strongly correlated electronic systems. Science, 2005, 309(5732): 257-262.

[9] TSAY C Y, HUANG Y H, HUNG D S. Enhanced microwave absorption of $\mathrm{La}_{0.7} \mathrm{Sr}_{0.3} \mathrm{MnO}_{3-\delta}$ based composites with added carbon black. Ceramics International, 2014, 40(3): 3947-3951.

[10] ZABALETA J, JAAFAR M,ABELLÁN P, et al. Nanoscale magnetic structure and properties of solution-derived self-assembled $\mathrm{La}_{0.7} \mathrm{Sr}_{0.3} \mathrm{MnO}_{3}$ islands. Journal of Applied Physics, 2012, 111(2): 024307-1-8.

[11] DATTA S,CHANDRA S,SAMANTA S, et al. Growth and physical property study of single nanowire (diameter $\sim 45 \mathrm{~nm}$ ) of half doped manganite. Journal of Nanomaterials, 2013, 2013: 162315-1-6.

[12] BANERJEE N, KRUPANIDHI S B. Anomalous magnetic behavior of $\mathrm{La}_{0.6} \mathrm{Sr}_{0.4} \mathrm{MnO}_{3}$ nano-tubes constituted with 3-12 nm particles. Applied Physics A, 2013, 111(2): 605-612.

[13] QI S Y, FENG J, XU X D, et al. Preparation and magnetic property of $\mathrm{La}_{0.7} \mathrm{Sr}_{0.3} \mathrm{MnO}_{3}$ nanorod by combination Sol-Gel with molten salt. Chemical Research in Chinese Universities, 2008, 24(6): 672-674.

[14] RUZMETOV D, SEO Y, BELENKY L J, et al. Epitaxial magnetic perovskite nanostructures. Advanced Materials, 2005, 17(23): 2869-2872.

[15] LIANG L Z, LI L, WU H, et al. Research progress on electronic phase separation in low-dimensional perovskite manganite nanostructures. Nanoscale Research Letters, 2014, 9(1): 325-1-14.

[16] ZHANG T, WANG X P, FANG Q F. Evolution of the electronic phase separation with magnetic field in bulk and nanometer $\mathrm{Pr}_{0.67} \mathrm{Ca}_{0.33} \mathrm{MnO}_{3}$ particles. Journal of Physical Chemistry C, 2011, 115(40): 19482-19487.

[17] LI L, LI H, ZHAI X F, et al. Fabrication and magnetic properties of single-crystalline $\mathrm{La}_{0.33} \mathrm{Pr}_{0.34} \mathrm{Ca}_{0.33} \mathrm{MnO}_{3} / \mathrm{MgO}$ nanowires. $A p$ plied Physics Letters, 2013, 103(11):113101-1-4.

[18] MOREO A, YUNOKI S, DAGOTTO E. Phase separation scenario for manganese oxides and related materials. Science, 1999, 283(5410): 2034-2040.

[19] YUNOKI S, MOREO A. Static and dynamical properties of the ferromagnetic Kondo model with direct antife fromagnetic coupling between the localized $\mathrm{t}_{2 \mathrm{~g}}$ electrons.Physical Review Letters, 1998, 58(10):6403-6413.

[20] YUNOKI S, MOREO A, DAGOTTO E. Phase separation induced by orbital degrees of freedom in models for manganites with Jahn-Teller phonons. Physical Review Letters, 1998, 81(25): $5612-5615$

[21] CHEN C H, CHEONG S W. Commensurate to incommensurate charge ordering and its real-space images in $\mathrm{La}_{0.5} \mathrm{Ca}_{0.5} \mathrm{MnO}_{3}$. Physical Review Letters, 1996, 76(21): 4042-4045. 
[22] RADAELI P G, COX D E, MAREZIO M, et al. Charge, orbital, and magnetic ordering in $\mathrm{La}_{0.5} \mathrm{Ca}_{0.5} \mathrm{MnO}_{3}$ s. Physical Review B, 1997, 55(5):3015-3023.

[23] MORI S, CHEN C H, CHEONG S W. Pairing of charge-ordered stripes in (La,Ca) $\mathrm{MnO}_{3}$. Nature, 1998, 392(6675): 473-476.

[24] ZHANG T, ZHOU T F, QIAN T, et al. Particle size effects on interplay between charge ordering and magnetic properties in nanosized $\mathrm{La}_{0.25} \mathrm{Ca}_{0.75} \mathrm{MnO}_{3}$. Physical Review B, 2007, 76: 174415-1-7.

[25] HUANG X H, DING J F, ZHANG G Q, et al. Size-dependent exchange bias in $\mathrm{La}_{0.25} \mathrm{Ca}_{0.75} \mathrm{MnO}_{3}$ nanoparticles. Physical Review B, 2008, 78: 224408-1-5.

[26] WANG Y F, YANG H, XIAN T, et al. Preparation and photocatalytic properties of $\mathrm{La}_{0.7} \mathrm{Sr}_{0.3} \mathrm{MnO}_{3}$ nanoparticles. Chinese Journal of Materials Research, 2012, 26(5): 476-482.

[27] JUGDERSUREN B, KANG S M, DIPIETRO R S, et al. Large low field magnetoresistance in $\mathrm{La}_{0.67} \mathrm{Sr}_{0.33} \mathrm{MnO}_{3}$ nanowire devices. $A p$ plied Physics Letters, 2011, 109(1): 016109-1-3.

[28] MARÍN L, MORELLÓN L, ALGARABEL P A, et al. Enhanced magnetotransport in nanopatterned manganite nanowires. Nano Letters, 2014, 14(2): 423-428.

[29] JIANG T, YANG S W, ZHOU H B, et al. Colossal anisotropic resistivity and oriented magnetic domains in strained $\mathrm{La}_{0.325} \mathrm{Pr}_{0.3} \mathrm{Ca}_{0.375} \mathrm{MnO}_{3}$ films. Applied Physics Letters, 2014, 104: 203501-1-5.

[30] NORI R,KALE S N, GANGULY U, et al. Morphology and Curie temperature engineering in crystalline $\mathrm{La}_{0.7} \mathrm{Sr}_{0.3} \mathrm{MnO}_{3}$ films on $\mathrm{Si}$ by pulsed laser deposition. Journal of Applied Physics, 2014,
115(3): 033518-1-11.

[31] HIROOKA M, YANAGISAWA Y, KANKI T, et al. Fabrication of sub-50 nm ( $\mathrm{La}, \mathrm{Ba}) \mathrm{MnO}_{3}$ ferromagnetic nanochannels by atomic force microscopy lithography and their electrical properties. Applied Physics Letters, 2006, 89(16): 163113-1-3.

[32] GUO X, LI P G, WANG X, et al. Anomalous positive magnetoresistance effect in $\mathrm{La}_{0.67} \mathrm{Ca}_{0.33} \mathrm{MnO}_{3}$ microbridges. Journal of Alloys and Compounds, 2009, 485(1/2): 802-806.

[33] KIM E J, WATTS J L R, HARTENECK B, et al. Magnetic domain structure of $\mathrm{La}_{0.7} \mathrm{Sr}_{0.3} \mathrm{MnO}_{3}$ nanoislands: experiment and simulation. Journal of Applied Physics, 2011, 109(7): 07D712.

[34] JIN K X, CHEN C L, ZHAO S G, et al. Spin transport properties of the thin film doped with $\mathrm{La}_{0.85} \mathrm{Sr}_{0.015} \mathrm{MnO}_{3}$. Rare Metal Materials And Engineering, 2007, 36(8): 1362-1365.

[35] TANG X Y, JIN K X, ZHANO S G, et al. The vacumm annealing effect on the photoinduced properties in $\mathrm{La}_{0.7} \mathrm{Sr}_{0.3} \mathrm{MnO}_{3}$ thin film. Chinese Journal of Material Research, 2009, 23(4): 395-398.

[36] LIU D Q, WANG N N,WANG G, et al. Programmable metallization cells based on amorphous $\mathrm{La}_{0.79} \mathrm{Sr}_{0.21} \mathrm{MnO}_{3}$ thin films for memory applications. Journal of Alloys and Compounds, 2013, 580: $354-357$.

[37] HOFFMAN J, HONG X, AHN C H. Device performance of ferroelectric/correlated oxide heterostructures for non-volatile memory applications. Nanotechnology, 2011, 22(25): 254014-1-4.

[38] HU M J, MA B W, WANG Z. Research on amperometric-type $\mathrm{NO}_{\mathrm{x}}$ sensor based on $\mathrm{La}_{1-x} \mathrm{Sr}_{x} \mathrm{MnO}_{3}$ sensitive materials. Chinese Journal of Analytical Chemistry, 2013, 41(10): 1531-1536. 